\title{
Assessment of sleep in children with periodic fever, aphthous stomatitis, pharyngitis, and adenitis syndrome
}

\author{
Ayşin Nalbantoğlu ${ }^{\oplus}$ Burçin Nalbantoğlu ${ }^{\odot}$ \\ Department of Pediatrics, Faculty of Medicine, Namık Kemal University Faculty of Medicine, Tekirda ̆̆, Turkey.
}

\begin{abstract}
Background. Periodic fever, aphthous stomatitis, pharyngitis and adenitis (PFAPA) syndrome is the most common cause of periodic fever in childhood. This study aimed to investigate sleep patterns and possible factors that are associated with sleep disturbances among children with PFAPA syndrome.
\end{abstract}

Methods. Sixty-two patients with PFAPA and 68 age and sex matched healthy controls were enrolled in the study. Patients who had an attack during the former 2 weeks were not included. Demographic and anthropometric data, duration of fever episodes, laboratory results, and clinical manifestations of patients were recorded. The Children's Sleep Habits Questionnaire was administered.

Results. The total sleep scores of patients with PFAPA were significantly higher than the control group (49.6 \pm 10.7 vs. $38.3 \pm 7.5, \mathrm{p}=0.002)$. Children with PFAPA had significantly higher scores regarding sleep-onset delay, sleep anxiety and night wakening $(\mathrm{p}=0.003, \mathrm{p}=0.007$, and $\mathrm{p}=0.014$, respectively). Total sleep durations were similar between children with PFAPA and the control group. There was a significant positive correlation between the total sleep score and disease duration $(\mathrm{r}=0.425, \mathrm{p}=0.002)$. Also there was a significant positive correlation between disease duration and sleep onset delay $(\mathrm{r}=0.561, \mathrm{p}<0.001)$ and night wakening $(\mathrm{r}=0.327$, $\mathrm{p}=0.003$ ).

Conclusion. This study showed for the first time that patients with PFAPA have significantly disturbed sleep when compared to otherwise normal children. This study emphasized the need to assess sleep problems in children with PFAPA

Key words: PFAPA, children, sleep, periodic fever.

Periodic fever, aphthous stomatitis, pharyngitis and adenitis (PFAPA) syndrome is the most common cause of periodic fever in childhood and generally seen among young children. ${ }^{1}$ Fever episodes occur in regular three to eight week cycles. However, the general condition of the patient is good, despite the high fever and other disease features. Between these episodes, patients are asymptomatic. Most patients have resolution of episodes until adolescence. ${ }^{2}$

凶 Ayşin Nalbantoğlu

analbantoglu@nku.edu.tr

Received 16th July 2020, revised 14th September 2020, 2nd October 2020, accepted 23rd December 2020.
The exact mechanism and etiology of PFAPA syndrome remains unclear. A dysregulation of the immune system or abnormal response to an unknown infection trigger is suspected. Recent studies support the hypothesis that PFAPA syndrome involves inflammasomedriven proinflammatory interleukin (IL)-1 $\beta$ production during febrile episodes. ${ }^{3}$ Acute phase parameters such as leukocyte counts, the erythrocyte sedimentation rate, C-reactive protein, and the excretion of proinflammatory cytokines increase during the fever episodes. ${ }^{4}$ Also hypovitaminosis $\mathrm{D}$ can be a significant risk factor for PFAPA recurrence. ${ }^{5}$ This strengthens the presence of underlying immune dysregulation. 
Sleep problems are much more prevalent among medically ill children when compared with the general population and the presence of co-morbidity may adversely affect medical outcomes and the quality of life of these patients. ${ }^{6}$ Sleep problems are important to assess due to both the potential for short and long term negative outcomes such as daytime behavioral problems, inattention, reduced academic performance and reduced health related quality of life. In previous studies, sleep problems have been described in several chronic rheumatological conditions such as juvenile dermatomyositis, familial Mediterranean fever (FMF), juvenile idiopathic arthritis (JIA), and systemic lupus erythematosus (SLE).$^{6-9}$ PFAPA shares many similar clinical features with monogenic auto-inflammatory recurrent fever syndromes, even though no genetic causes of PFAPA have been documented. PFAPA is generally considered to be a benign disease, however fever attacks have been shown to have a substantial impact on daily activities, school and family functioning. ${ }^{10}$ There is only one study in the literature demonstrating that the well-being of children with PFAPA is poor, with a major impact on psychosocial functioning and increased fatigue. ${ }^{11}$ Previous studies show that sleep disturbances may be associated with inflammation and inflammatory mediators effect the regulation of sleep in the central nervous system..$^{6-9}$ Therefore as an inflammatory disease, sleep is a prominent issue, even though this has not yet been studied in PFAPA patients. The aim of this study is to assess the sleep habits of children and possible factors that are associated with sleep disturbances using an objective and reliable tool among children with PFAPA syndrome.

\section{Material and Methods}

This study was conducted on patients recruited from the out-patient clinic of our institute, between 1 February 2020 and 1 May 2020. We consecutively evaluated 62 patients aged between 4-8 years, after having excluded other causes of recurrent fever. All patients were enrolled in the study during an attack free period. Hence patients who had an attack during the former 2 weeks were not included. The diagnosis of PFAPA was made according to international criteria suggested by Thomas et al. ${ }^{12}$ We recorded demographic and anthropometric data, duration of fever episodes, laboratory results, and clinical manifestations of patients. Exclusion criteria from the study were; patients who had infections, any chronic or psychiatric diseases other than PFAPA, and any chronic drug use. None of the patients had enlarged tonsils, adenoid problem or morbid obesity, which may cause obstructive apnea.

The control group included 68 patients aged between 4-8 years without PFAPA history and were recruited from the children who visited the well child outpatient clinic of our department. Demographic data of the control group was also collected. The control group were matched for age, sex, economic status and parental education with the patient group. The control group had no history of any sleep disorders or used any medication that could cause sleep problems.

Tekirdağ Namık Kemal University Ethics Committee approved the study protocol (25/02/2020-2020.35.02.09). All legal guardians of patients were informed about the scale and written informed consent was received.

\section{Children's Sleep Habits Questionnaire}

The Children's Sleep Habits Questionnaire (CSHQ) is a parent-report instrument that was used to measure the external validity of the family inventory of sleep habits and assess the typical sleep patterns of preschool and schoolage children. ${ }^{13,14}$ The $\mathrm{CSQH}$ is a retrospective, 33-item parent questionnaire that has been used in a number of studies to examine sleep behaviors in children, and appears to have adequate validity and reliability. The validity and the reliability of the Turkish version of the CSHQ has been previously shown. ${ }^{15}$ Parents are asked to recall sleep behaviors occurring over a typical recent week. Items are rated on a 3-point 
scale for frequency of the sleep behavior: usually, 5-7 times/week; sometimes, 2-4 times/ week; and rarely, 0-1 times/week. Scores are adjusted so that a higher score was indicative of more disturbed sleep. In addition to a total score, 33 items of the CSHQ were grouped into the following 8 subscales: (1) bedtime resistance, (2) sleep onset delay, (3) sleep duration, (4) sleep anxiety, (5) sleep-disordered breathing, (6) night wakening, (7) parasomnias, and (8) morning wakening/daytime sleepiness. ${ }^{15}$ If the patients have an unusual event in the week prior to the questionnaire, parents were asked to fill it according to the nearest typical week. A total $\mathrm{CSQH}$ score of higher than 41 has been reported to be a sensitive clinical cut off for screening purposes and identification of probable sleep problems. ${ }^{14}$

\section{Statistical analysis}

Data were analyzed using SPSS 21 (IBM SPSS, Chicago, IL, USA). Kolmogorov-Smirnov test was used to check for whether the data was normally or non-normally distributed. Nonnormally distributed data were expressed as median and minimum-maximum values; normally distributed data were expressed as the mean \pm standard deviation. Student's t-test and Mann-Whitney $U$ test were used as appropriate. The Chi-square test was used to test differences in categorical variables between the two groups. Intercorrelations between parameters were computed through Pearson's correlation analysis. $P$ values $<0.5$ was accepted as significant. Internal consistency of the scale was determined by calculating Cronbach's coefficient alpha. Scales with reliabilities of 0.70 or greater are recommended for comparing groups.

\section{Results}

Table I shows the demographic data of the groups. The total sleep scores of the patients with PFAPA were significantly higher than the control group $(49.6 \pm 10.7$ vs. $38.3 \pm 7.5, \mathrm{p}$ $=0.002)$. In patient group $41(66.1 \%)$ patients had $\mathrm{CSQH}$ scores higher than 41 (clinically significant sleep disturbance), whereas in the control group 23 (\%33.8) had CSQH scores higher than $41(\mathrm{p}=0.001)$. Children with PFAPA had significantly higher scores regarding sleeponset delay, sleep anxiety and night wakening ( $\mathrm{p}=0.003, \mathrm{p}=0.007$, and $\mathrm{p}=0.014$, respectively) (Table II). Total sleep durations were comparable between children with PFAPA and the control group $(8.7 \pm 1.7$ vs. $9.0 \pm 1.2$ hours, $\mathrm{p}=0.36$ ). The total sleep scores of the male PFAPA patients were significantly higher than the female PFAPA patients $(51.1 \pm 9.9$ vs. $46.4 \pm$ 9.6; $\mathrm{p}=0.025$ ).

There was no significant correlation between the total sleep score and number of attacks in the last year $(\mathrm{r}=0.006, \mathrm{p}=0.54)$. There was a significant positive correlation between the total sleep score and disease duration $(\mathrm{r}=0.425$, $\mathrm{p}=0.002)$. Also there was a significant positive correlation between disease duration and sleep onset delay $(\mathrm{r}=0.561, \mathrm{p}<0.001)$, and night wakening $(\mathrm{r}=0.327, \mathrm{p}=0.003)$.

Internal consistency reliability alpha coefficient for CSHQ in the patient group and in the control group were 0.79 and 0.72 , respectively.

Table I. Demographic data of patient and control groups.

\begin{tabular}{|c|c|c|c|}
\hline Characteristics & PFAPA group $(\mathrm{N}=62)$ & Control group $(\mathrm{N}=68)$ & $P$ value \\
\hline Sex (male), n (\%) & $35(56.5 \%)$ & $35(51.5 \%)$ & 0.694 \\
\hline Age (years) & $5.8 \pm 2.1$ & $6.1 \pm 2.2$ & 0.752 \\
\hline Duration of fever (days) & $5(0.7-11)$ & & \\
\hline Time between the attacks (week) & $7(1.3-10)$ & & \\
\hline Disease duration (years) & $4.8(1.1-6.8)$ & & \\
\hline
\end{tabular}

Continuous variables are presented as mean \pm standard deviation or median (minimum - maximum), as appropriate. 
Table II. Sleep parameters of patient and control groups.

\begin{tabular}{lccc}
\hline Parameters & PFAPA group $(\mathrm{N}=62)$ & Control group $(\mathrm{N}=68)$ & P value \\
\hline Total sleep duration (hours) & $8.7 \pm 1.7$ & $9.0 \pm 1.2$ & 0.360 \\
Total sleep score & $49.6 \pm 10.7$ & $38.3 \pm 7.5$ & 0.002 \\
Sleep score $>41$ & $41(66.1 \%)$ & $23(33.8 \%)$ & 0.001 \\
Subscales & & & \\
$\quad$ Bedtime resistance & $7.7 \pm 3.9$ & $8.1 \pm 3.2$ & 0.067 \\
Sleep-onset delay & $2.7 \pm 1.3$ & $1.6 \pm 0.9$ & 0.001 \\
Sleep duration & $4.1 \pm 1.7$ & $4.2 \pm 1.9$ & 0.765 \\
Sleep anxiety & $6.5 \pm 2.5$ & $4.9 \pm 1.9$ & 0.001 \\
Night wakening & $4.8 \pm 1.2$ & $3.7 \pm 1.4$ & 0.002 \\
$\quad$ Parasomnias & $8.3 \pm 2.3$ & $8.8 \pm 2.4$ & 0.923 \\
Sleep-disordered breathing & $3.8 \pm 1.5$ & $3.6 \pm 0.9$ & 0.628 \\
Day-time sleepiness & $14.8 \pm 4.8$ & $14.1 \pm 3.8$ & 0.743 \\
\hline
\end{tabular}

Data are presented as mean \pm standard deviation or $\mathrm{n}(\%)$.

\section{Discussion}

The results of this study indicated that patients with PFAPA had more sleep problems than their healthy peers. The patients with PFAPA reported higher scores of sleep-onset delay, sleep anxiety and night wakening, compared to healthy controls. Clinically significant sleep disturbance rate was higher in PFAPA syndrome ( $66.1 \%$ vs $33.8 \%$; $\mathrm{p}=0.001)$. Also, there was a significant positive correlation between the total sleep score and disease duration. To the best of our knowledge, this is the first study investigating sleep problems among children with PFAPA.

Inflammatory cytokines can affect the regulation of sleep in the central nervous system and sleep may play a vital role in immune regulation. ${ }^{15}$ Many inflammatory cytokines such as IL-1 $\beta$ and IL-6 were elevated either during or between attacks in PFAPA patients. ${ }^{16}$ IL-6, a proinflammatory cytokine, elevation was shown to affect the organism, with a focus on sleep-related symptoms and fatigue. ${ }^{17}$ As a result of this data, we are of the opinion that inflammation can contribute to sleep disturbance of PFAPA patients.

Makay et al. $^{6}$ conducted a study assessing sleep problems in children with FMF using CSHQ. They found significantly higher scores regarding sleep-onset delay, sleep anxiety, night wakening and sleep-disordered breathing in patients with FMF when compared to healthy controls. The findings of our study is similar to their results, but we found no relationship between number of attacks per year and sleep scores unlike their study. Also, in our study, there was a significant positive correlation between the total sleep score and disease duration. We found no correlation between sleep disordered breathing and PFAPA. Despite the diversity of clinical presentation, there are some common features between PFAPA and FMF. ${ }^{1}$ In some of the PFAPA patients MEFV gene mutation may be present similar to FMF. ${ }^{20}$ Potential prophylactic benefits of colchicine reported in the literature supports a similar immunological basis for PFAPA and FMF. ${ }^{21}$ Also, sleep problems have been described in several chronic rheumatological conditions such as juvenile dermatomyositis, FMF, JIA, and SLE..$^{6-9}$ In a study conducted by Bloom et al. ${ }^{16}$, patients with JIA had higher total score on CSHQ, as well as subscales assessing night wakening, parasomnias, sleep anxiety, sleepdisordered breathing, and morning wakening/ daytime sleepiness.

In a previous study by Grimwood et al. ${ }^{11}$ investigating the health related quality of life (HRQOL) in children with PFAPA syndrome, 
they showed that the wellbeing of PFAPA children is poor, with a major impact on psychosocial functioning and increased fatigue. They found the quality of life of PFAPA children to be even lower than that of FMF patients, for whom a lower than normal HRQOL has already been demonstrated. Although PFAPA is known as a self-limited and benign condition, it may have major impact on HRQOL and sleep patterns in both patients and their parents as suggested by our study.

Gender differences in total sleep scores were observed in our cohort, boys had higher scores than girls which is consistent with previous studies. ${ }^{22,23}$ An explanation for the gender difference is unclear; however, it is possible that girls may require more sleep than boys, that parenting practices or social obligations may vary, or that girls may be more attentive to their sleep needs. ${ }^{23}$

Adequate sleep in young children is important for both healthy development and optimum daytime functioning. Sleep problems in young children may be problematic for parents, but such problems may be related to additional problems for the children both concurrently and over time. ${ }^{24}$ Increased sleep problems have been related to increased internalizing and externalizing problems, increased injury rates and although many children experience improvements with sleep over time, for a substantial subset of children who exhibits sleep disturbances at a young age are more likely to exhibit persistent sleep problems. ${ }^{23}$ Persistent sleep problems are more likely to have other difficulties over time such as negative behavior and poor academic skills.

Parents' education regarding what is adequate sleep hygiene may be the start for treatment in PFAPA patients. Physical activity, which, when moderate, has a beneficial effect on sleep. At least 3 hours before the child's established bedtime, he/she should be involved in relaxing activities; over stimulation should be avoided. Activities involving electronic media (TV, computer, tablet, and mobile phone) should also be restricted and avoided at least 1 hour before bedtime. The bed room environment is also a sleep hygiene factor. It should be well ventilated, quiet and dark, at an adequate temperature, and have a comfortable bed. Positive routines can also help the child learn appropriate sleep behaviors and reduce stress. In addition to defining bedtime, consistent routines (activities that help prepare for sleep) should be established and repeated every night. Brushing the teeth, performing other hygiene routines, putting on pajamas, reading a story or spending some time with parents, turning off the lights could be given as examples. It is important to make sure that the time set for the routine is sufficient, so that they can be conducted calmly, without harming total sleep time. ${ }^{25,26}$ Despite these interventions, if the problem still continues, patient may be referred to a sleep expert.

The limitations of this study is the small sample size and the inclusion of children who were attending the same clinic, which may limit the generalizing the results of this study to all children with PFAPA. As diagnostic limitations we did not perform a polysomnography and the data for sleep disturbances was collected by a questionnaire, but owing the reason that our study is the first work on this topic, we think it is still worth sharing. The strength of this study includes the use of a pediatric sleep habit questionnaire with previously demonstrated reliability and validity in pediatric rheumatic diseases.

The present study underlines the need to assess and manage sleep problems in children with PFAPA for the first time. Our results showed that patients with PFAPA have significantly disturbed sleep when compared to otherwise normal children. Given the sleep problems in children with PFAPA, as well as the potential for negative consequences associated with sleep problems, it is important to be able to identify and intervene with sleep problems at an early 
age. Poor sleep in children has been associated with family stress and health issues as well. The results of this study to assess the sleep problems of PFAPA children refutes the belief that PFAPA is a benign disease and demonstrates that the consequences of PFAPA has probably been largely underestimated. To our opinion, sleep habits of PFAPA patients must be questioned, and every effort including sleep hygiene techniques should be made to resolve sleep problems, in order to avoid long term negative consequences. However, further studies using polysomnography with larger sample sizes and from different centers are needed to confirm this hypothesis.

\section{Author contribution}

The authors confirm contribution to the paper as follows: study conception and design: $\mathrm{AN}, \mathrm{BN}$; data collection: $\mathrm{AN}$; analysis and interpretation of results: $\mathrm{AN}, \mathrm{BN}$; draft manuscript preparation: AN, BN. All authors reviewed the results and approved the final version of the manuscript.

\section{Ethical approval}

Tekirdağ Namık Kemal University Ethics Committee approved the study protocol (25/02/2020-2020.35.02.09).

\section{Source of funding}

No funding was received.

\section{Conflict of interest}

Author A. Nalbantoğlu and B. Nalbantoğlu declare that they have no conflict of interest.

\section{REFERENCES}

1. Adrovic A, Sahin S, Barut K, Kasapcopur O. Familial mediterrenean fever and periodic fever, aphthous stomatitis, pharyngitis, and adenitis (PFAPA) syndrome: shared features and main differences. Rheumatol Int 2019; 39: 29-36.
2. Manthiram K, Correa H, Boyd K, Roland J, Edwards $\mathrm{K}$. Unique histologic features of tonsils from patients with periodic fever, aphthous stomatitis, pharyngitis, and cervical adenitis (PFAPA) syndrome. Clin Rheumatol 2018; 37: 1309-1317.

3. Kolly L, Busso N, von Scheven-Gete A, et al. Periodic fever, aphthous stomatitis, pharyngitis, cervical adenitis syndrome is linked to dysregulated monocyte IL- $1 \beta$ production. J Allergy Clin Immunol 2013; 131: 1635-1643.

4. Tejesvi MV, Uhari M, Tapiainen T, et al. Tonsillar microbiota in children with PFAPA (periodic fever, aphthous stomatitis, pharyngitis, and adenitis) syndrome. Eur J Clin Microbiol Infect Dis 2016; 35: 963-970.

5. Nalbantoglu A, Nalbantoglu B. Vitamin D deficiency as a risk factor for PFAPA syndrome. Int J Pediatr Otorhinolaryngol 2019; 121: 55-57.

6. Makay B, Kılıçaslan SK, Anık A, et al. Assessment of sleep problems in children with familial Mediterranean fever. Int J Rheum Dis 2017; 20: 21062112.

7. Kucuksahin O, Omma A, Ozdemirel AE, et al. Incidence of sleep disturbances in patients with familial Mediterranean fever and the relation of sleep quality with disease activity. Int J Rheum Dis 2018; 21: 1849-1856.

8. Butbul Aviel Y, Stremler R, Benseler SM et al. Sleep and fatigue and the relationship to pain, disease activity and quality of life in juvenile idiopathic arthritis and juvenile dermatomyositis. Rheumatology (Oxford) 2011; 50: 2051-2060.

9. Vina ER, Green SL, Trivedi T, Kwoh CK, Utset TO. Correlates of sleep abnormalities in systemic lupus: a cross-sectional survey in an urban, academic center. J Clin Rheumatol 2013; 19: 7-13.

10. Feder HM, Salazar JC. A clinical review of 105 patients with PFAPA (a periodic fever syndrome). Acta Paediatr 2010; 99: 178-184.

11. Grimwood C, Kone-Paut I, Piram M, RossiSemerano L, Hentgen V. Health-related quality of life in children with PFAPA syndrome. Orphanet J Rare Dis 2018; 13: 132.

12. Thomas KT, Edwards KM. Periodic fever syndrome. Pediatr Infect Dis J 1999; 18: 68-69.

13. Malow BA, Crowe C, Henderson L, et al. A sleep habits questionnaire for children with autism spectrum disorders. J Chil Neurol 2009; 24: 19-24.

14. Owens JA, Spirito A, McGuinn M. The Children's Sleep Habits Questionnaire (CSHQ): psychometric properties of a survey instrument for school-aged children. Sleep 2000; 23: 1043-1051. 
15. Perdahlı Fiş N, Arman A, Ay P, et al. Çocuk uyku alışkanlıkları anketinin Türkçe geçerliliği ve güvenilirliği. Anadolu Psikiyatri Dergisi 2010; 11: 151-160.

16. Bloom BJ, Owens JA, McGuinn M, Nobile C, Schaeffer L, Alario AJ. Sleep and its relationship to pain, dysfunction, and disease activity in juvenile rheumatoid arthritis. J Rheumatol 2002; 29: 169-173.

17. Boakye PA, Olechowski C, Rashiq S, et al. A critical review of neurobiological factors involved in the interactions between chronic pain, depression, and sleep disruption. Clin J Pain 2016; 32: 327-336.

18. Stojanov S, Hoffmann F, Kery A, et al. Cytokine profile in PFAPA syndrome suggests continous inflammation and reduced anti-inflammatory response. Eur Cytokine Netw 2006; 17: 90-97.

19. Rohleder N, Aringer M, Boentert M. Role of interleukin-6 in stress, sleep, and fatigue. Ann N Y Acad Sci 2012; 1261: 88-96.

20. Pehlivan E, Adrovic A, Sahin S, Barut K, Kul Cınar O, Kasapcopur O. PFAPA syndrome in a population with endemic familial Mediterranean fever. J Pediatr 2018; 192: 253-255.

21. Tasher D, Stein M, Dalal I, Somekh E. Colchicine prophylaxis for frequent periodic fever, aphthous stomatitis pharyngitis and adenitis episodes. Acta Paediatr 2008; 97: 1090-1092.
22. Biggs SN, Lushington $K$, James Martin A, van den Heuvel C, Declan Kennedy J. Gender, socioeconomic and ethnic differences in sleep patterns in schoolaged children. Sleep Med2013; 14: 1304-1309.

23. Blair PS, Humphreys JC, Gringras P, et al. Childhood sleep duration and associated demographic characteristics in an English cohort. Sleep 2012; 35: 353-360.

24. Sneddon P, Peacock GG, Crowley SL. Assessment of sleep problems in preschool aged children: an adaptation of the children's sleep habits questionnaire. Behav Sleep Med2013; 11: 283-296.

25. Thome M, Skuladottir A. Changes in sleep problems, parents distress and impact of sleep problems from infancy to preschool age for referred and unreferred children. Scand J Caring Sci 2005; 19: 86-94.

26. Nunes ML, Bruni O. Insomnia in childhood and adolescence: clinical aspects, diagnosis, and theurapeutic approach. J Pediatr (Rio J) 2015; 91 (6 Suppl 1): S26-S35. 